\title{
Global holomorphic functions in several non-commuting variables II
}

\author{
Jim Agler * \\ U.C. San Diego \\ La Jolla, CA 92093
}

\author{
John E. McCarthy ${ }^{\dagger}$ \\ Washington University \\ St. Louis, MO 63130
}

September 13, 2018

\begin{abstract}
We give a new proof that bounded non-commutative functions on polynomial polyhedra can be represented by a realization formula, a generalization of the transfer function realization formula for bounded analytic functions on the unit disk.

Math Subject Classification: 15A54
\end{abstract}

\section{Introduction}

Let $\mathbb{M}_{n}$ denote the $n$-by- $n$ matrices with complex entries, and let $\mathbb{M}^{d}=$ $\cup_{n=1}^{\infty} \mathbb{M}_{n}^{d}$ be the set of all $d$-tuples of matrices of the same size. An ncfunction ${ }^{1}$ on a set $E \subseteq \mathbb{M}^{d}$ is a function $\phi: E \rightarrow \mathbb{M}^{1}$ that satisfies

(i) $\phi$ is graded, which means that if $x \in E \cap \mathbb{M}_{n}^{d}$, then $\phi(x) \in \mathbb{M}_{n}$.

(ii) $\phi$ is intertwining preserving, which means if $x, y \in E$ and $S$ is a linear operator satisfying $S x=y S$, then $S \phi(x)=\phi(y) S$.

The points $x$ and $y$ are $d$-tuples, so we write $x=\left(x^{1}, \ldots, x^{d}\right)$ and $y=$ $\left(y^{1}, \ldots, y^{d}\right)$. By $S x=y S$ we mean that $S x^{r}=y^{r} S$ for each $1 \leq r \leq d$. See [9] for a general reference to nc-functions.

\footnotetext{
*Partially supported by National Science Foundation Grants DMS 1361720 and DMS 1665260

${ }^{\dagger}$ Partially supported by National Science Foundation Grant DMS 1565243

${ }^{1} \mathrm{nc}$ is short for non-commutative
} 
The principal result of [2] was a realization formula for nc-functions that are bounded on polynomial polyhedra; the object of this note is to give a simpler proof of this formula, Theorem 1.5 below.

Let $\delta$ be an $I$-by- $J$ matrix whose entries are non-commutative polynomials in $d$-variables. If $x \in \mathbb{M}_{n}^{d}$, then $\delta(x)$ can be naturally thought of as an element of $\mathcal{B}\left(\mathbb{C}^{J} \otimes \mathbb{C}^{n}, \mathbb{C}^{I} \otimes \mathbb{C}^{n}\right)$, where $\mathcal{B}$ denotes the bounded linear operators, and all norms we use are operator norms on the appropriate spaces. We define

$$
B_{\delta}:=\left\{x \in \mathbb{M}^{d}:\|\delta(x)\|<1\right\} .
$$

Any set of the form (1.1) is called a polynomial polyhedron. Let $H^{\infty}\left(B_{\delta}\right)$ denote the nc-functions on $B_{\delta}$ that are bounded, and $H_{1}^{\infty}\left(B_{\delta}\right)$ denote the closed unit ball, those nc-functions that are bounded by 1 for every $x \in B_{\delta}$.

Definition 1.2. A free realization for $\phi$ consists of an auxiliary Hilbert space $\mathcal{M}$ and an isometry

$$
\begin{aligned}
& \mathbb{C} \\
& \mathbb{M} \otimes \mathbb{C}^{J}\left(\begin{array}{cc}
A & B \\
C & D
\end{array}\right)
\end{aligned}
$$

such that, for all $x \in B_{\delta}$, we have

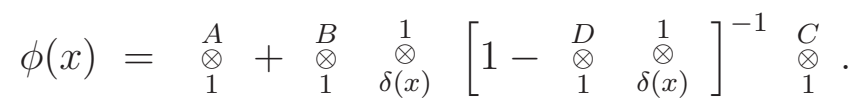

The 1's need to be interpreted appropriately. If $x \in \mathbb{M}_{n}^{d}$, then (1.4) means

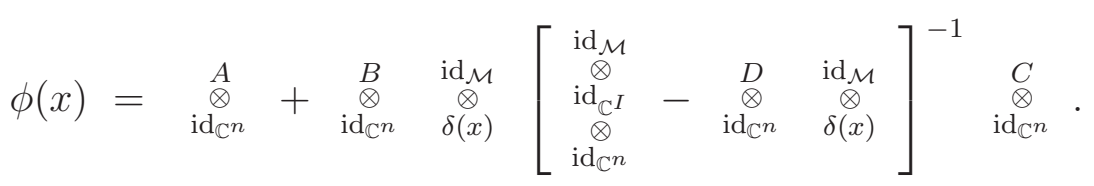

We adopt the convention of [11] and write tensors vertically to enhance legibility. The bottom-most entry corresponds to the space on which $x$ originally acts; the top corresponds to the intrinsic part of the model on $\mathcal{M}$.

The following theorem was proved in [2]; another proof appears in [6].

Theorem 1.5. The function $\phi$ is in $H_{1}^{\infty}\left(B_{\delta}\right)$ if and only if it has a free realization.

It is a straightforward calculation that any function of the form (1.4) is in $H_{1}^{\infty}\left(B_{\delta}\right)$. We wish to prove the converse. We shall use two other results, Theorems 1.8 and 1.9 below.

If $E \subset \mathbb{M}^{d}$, we let $E_{n}$ denote $E \cap \mathbb{M}_{n}^{d}$. If $\mathcal{K}$ and $\mathcal{L}$ are Hilbert spaces, a $\mathcal{B}(\mathcal{K}, \mathcal{L})$-valued nc function on a set $E \subseteq \mathbb{M}^{d}$ is a function $\phi$ such that 
(i) $\phi$ is $\mathcal{B}(\mathcal{K}, \mathcal{L})$ graded, which means if $x \in E_{n}$, then $\phi(x) \in \mathcal{B}\left(\mathcal{K} \otimes \mathbb{C}^{n}, \mathcal{L} \otimes\right.$ $\left.\mathbb{C}^{n}\right)$.

(ii) $\phi$ is intertwining preserving, which means if $x, y \in E$ and $S$ is a linear operator satisfying $S x=y S$, then

$$
\underset{S}{\stackrel{\operatorname{id}_{\mathcal{L}}}{\otimes}} \phi(x)=\phi(y) \underset{S}{\stackrel{\otimes}{\operatorname{id} \mathcal{N}}}
$$

Definition 1.6. An nc-model for $\phi \in H_{1}^{\infty}\left(B_{\delta}\right)$ consists of an auxiliary Hilbert space $\mathcal{M}$ and a $\mathcal{B}\left(\mathbb{C}, \mathcal{M} \otimes \mathbb{C}^{J}\right)$-valued nc-function $u$ on $B_{\delta}$ such that, for all pairs $x, y \in B_{\delta}$ that are on the same level (i.e. both in $B_{\delta} \cap \mathbb{M}_{n}^{d}$ for some $n$ )

$$
1-\phi(y)^{*} \phi(x)=u(y)^{*}\left[\begin{array}{c}
\stackrel{1}{\otimes} \\
1-\delta(y)^{*} \delta(x)
\end{array}\right] u(x) .
$$

Again, the 1's have to be interpreted appropriately. If $x, y \in B_{\delta} \cap \mathbb{M}_{n}^{d}$, then (1.7) means

$$
\operatorname{id}_{\mathbb{C}^{n}}-\phi(y)^{*} \phi(x)=u(y)^{*}\left[\begin{array}{c}
\stackrel{\mathrm{id}_{\mathcal{M}}}{\otimes} \\
\operatorname{id}_{\mathbb{C}^{J} \otimes \mathbb{C}^{n}}-\delta(y)^{*} \delta(x)
\end{array}\right] u(x) .
$$

Theorem 1.8. A graded function on $B_{\delta}$ has an nc-model if and only if it has a free realization.

Theorem 1.8 was proved in [2], but a simpler proof is given by S. Balasubramanian in [5]. Let us note for future reference that the functions $u$ in (1.7) are locally bounded, and therefore holomorphic [2, Thm. 4.6].

The finite topology on $\mathbb{M}^{d}$ (also called the disjoint union topology) is the topology in which a set $\Omega$ is open if and only if for every $n, \Omega_{n}$ is open in the Euclidean topology on $\mathbb{M}_{n}^{d}$. If $\mathcal{H}$ is a Hilbert space, and $\Omega$ is finitely open, we shall let $\operatorname{Hol}_{\mathcal{H}}^{\text {nc }}(\Omega)$ denote the $\mathcal{B}(\mathbb{C}, \mathcal{H})$ graded nc-functions on $\Omega$ that are holomorphic on each $\Omega_{n}{ }^{2}$ A sequence of functions $u^{k}$ on $\Omega$ is finitely locally uniformly bounded if for each point $\lambda \in \Omega$, there is a finitely open neighborhood of $\lambda$ inside $\Omega$ on which the sequence is uniformly bounded.

The following wandering Montel theorem is proved in [1]. If $u$ is in $\operatorname{Hol}_{\mathcal{H}}^{\text {nc }}(\Omega)$ and $V$ is a unitary operator on $\mathcal{H}$, define $V * u$ by

$$
\left.\forall_{n} \quad(V * u)\right|_{\Omega_{n}}=\left.\underset{\operatorname{id}_{\mathbb{C}^{n}}}{\stackrel{V}{\otimes}} u\right|_{\Omega_{n}} .
$$

\footnotetext{
${ }^{2} \mathrm{~A}$ function $u$ is holomorphic in this context if for each $n$, for each $x \in \Omega_{n}$, for each $h \in \mathbb{M}_{n}^{d}$, the limit $\lim _{t \rightarrow 0} \frac{1}{t}(u(x+t h)-u(x))$ exists.
} 
Theorem 1.9. Let $\Omega$ be finitely open, $\mathcal{H}$ a Hilbert space, and $\left\{u^{k}\right\}$ a finitely locally uniformly bounded sequence in $\operatorname{Hol}_{\mathcal{H}}^{\text {nc }}(\Omega)$. Then there exists a sequence $\left\{U^{k}\right\}$ of unitary operators on $\mathcal{H}$ such that $\left\{U^{k} * u^{k}\right\}$ has a subsequence that converges finitely locally uniformly to a function in $\operatorname{Hol}_{\mathcal{H}}^{\text {nc }}\left(B_{\delta}\right)$.

Let $\phi \in H_{1}^{\infty}\left(B_{\delta}\right)$. We shall prove Theorem 1.5 in the following steps.

I For every $z \in B_{\delta}$, show that $\phi(z)$ is in $\operatorname{Alg}(z)$, the unital algebra generated by the elements of $z$.

II Prove that for every finite set $F \subseteq B_{\delta}$, there is an nc-model for a function $\psi$ that agrees with $\phi$ on $F$.

III Show that these nc-models have a cluster point that gives an nc-model for $\phi$.

IV Use Theorem 1.8 to get a free realization for $\phi$.

Remarks:

1. Step I is noted in [2] as a corollary of Theorem 1.5; proving it independently allows us to streamline the proof of Theorem 1.5.

2. To prove Step II, we use one direction of [3, Thm 1.3] that gives necessary and sufficient conditions to solve a finite interpolation problem on $B_{\delta}$. The proof of necessity of this theorem used Theorem 1.5 above, but for Step II we only need the sufficiency of the condition, and the proof of this in [3] did not use Theorem 1.5.

3. All three known proofs of Theorem 1.5 start by proving a realization on finite sets, and then somehow taking a limit. In [2], this is done by considering partial nc-functions; in [6], it is done by using noncommutative kernels to get a compact set in which limit points must exist; in the current paper, we use the wandering Montel theorem.

\section{Step I}

Let $\left\{e_{j}\right\}_{j=1}^{n}$ be the standard basis for $\mathbb{C}^{n}$. For $x$ in $\mathbb{M}_{n}$ or $\mathbb{M}_{n}^{d}$, let $x^{(k)}$ denote the direct sum of $k$ copies of $x$. If $x \in \mathbb{M}_{n}^{d}$ and $s$ is invertible in $\mathbb{M}_{n}$, then $s^{-1} x s$ denotes the $d$-tuple $\left(s^{-1} x^{1} s, \ldots, s^{-1} x^{d} s\right)$. 
Lemma 2.1. Let $z \in \mathbb{M}_{n}^{d}$, with $\|z\|<1$. Assume $w \notin \operatorname{Alg}(z)$. Then there is an invertible $s \in \mathbb{M}_{n^{2}}$ such that $\left\|s^{-1} z^{(n)} s\right\|<1$ and $\left\|s^{-1} w^{(n)} s\right\|>1$.

Proof: Let $\mathcal{A}=\operatorname{Alg}(z)$. Since $w \notin \mathcal{A}$, and $\mathcal{A}$ is finite dimensional and therefore closed, the Hahn-Banach theorem says that there is a matrix $K \in \mathbb{M}_{n}$ such that $\operatorname{tr}(a K)=0 \forall a \in \mathcal{A}$ and $\operatorname{tr}(w K) \neq 0$. Let $u \in \mathbb{C}^{n} \otimes \mathbb{C}^{n}$ be the direct sum of the columns of $K$, and $v=e_{1} \oplus e_{2} \oplus \ldots e_{n}$. Then for any $b \in \mathbb{M}_{n}$ we have

$$
\operatorname{tr}(b K)=\left\langle b^{(n)} u, v\right\rangle .
$$

Let $\mathcal{A} \otimes$ id denote $\left\{a^{(n)}: a \in \mathcal{A}\right\}$. We have $\left\langle a^{(n)} u, v\right\rangle=0 \forall a \in \mathcal{A}$ and $\left\langle w^{(n)} u, v\right\rangle \neq 0$.

Let $\mathcal{N}=(\mathcal{A} \otimes \mathrm{id}) u$. This is an $\mathcal{A} \otimes$ id-invariant subspace, but it is not $w^{(n)}$ invariant (since $v \perp \mathcal{N}$, but $v$ is not perpendicular to $w^{(n)} u$ ). So decomposing $\mathbb{C}^{n} \otimes \mathbb{C}^{n}$ as $\mathcal{N} \oplus \mathcal{N}^{\perp}$, every matrix in $\mathcal{A} \otimes$ id has 0 in the $(2,1)$ entry, and $w^{(n)}$ does not.

Let $s=\alpha I_{\mathcal{N}}+\beta I_{\mathcal{N}^{\perp}}$, with $\alpha>\beta>0$. Then

$$
s^{-1}\left[\begin{array}{cc}
A & B \\
C & D
\end{array}\right] s=\left[\begin{array}{cc}
A & \frac{\beta}{\alpha} B \\
\frac{\alpha}{\beta} C & D
\end{array}\right] .
$$

If the ratio $\alpha / \beta$ is large enough, then for each of the $d$ matrices $z^{r}$, the corresponding $s^{-1}\left(z^{r} \otimes \mathrm{id}\right) s$ will have strict contractions in the $(1,1)$ and $(2,2)$ slots, and each $(1,2)$ entry will be small enough so that the whole thing is a contraction.

For $w$, however, as the $(2,1)$ entry is non-zero, the norm of $s^{-1} w^{(n)} s$ can be made arbitrarily large.

Lemma 2.2. Let $z \in B_{\delta} \cap \mathbb{M}_{n}^{d}$, and $w \in \mathbb{M}_{n}$ not be in $\mathcal{A}:=\operatorname{Alg}(z)$. Then there is an invertible $s \in \mathbb{M}_{n^{2}}$ such that $s^{-1} z^{(n)} s \in B_{\delta}$ and $\left\|s^{-1} w^{(n)} s\right\|>1$.

Proof: As in the proof of Lemma 2.1, we can find an invariant subspace $\mathcal{N}$ for $\mathcal{A} \otimes$ id that is not $w$-invariant. Decompose $\delta\left(z^{(n)}\right)$ as a map from $\left(\mathcal{N} \otimes \mathbb{C}^{J}\right) \oplus\left(\mathcal{N}^{\perp} \otimes \mathbb{C}^{J}\right)$ into $\left(\mathcal{N} \otimes \mathbb{C}^{I}\right) \oplus\left(\mathcal{N}^{\perp} \otimes \mathbb{C}^{I}\right)$. With $s$ as in Lemma 2.1, and $\alpha>>\beta>0$, and $P$ the projection from $\mathbb{C}^{n} \otimes \mathbb{C}^{n}$ onto $\mathcal{N}$, we get

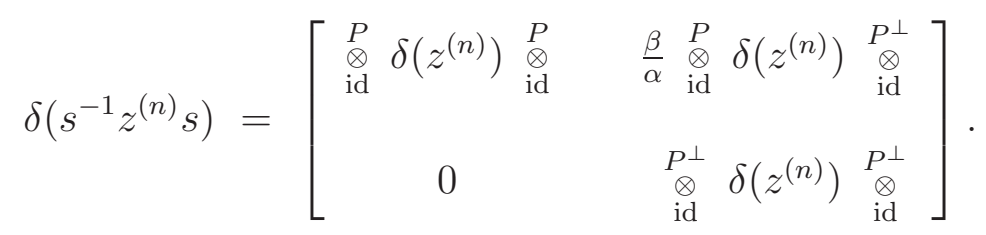


The matrix is upper triangular because every entry of $\delta$ is a polynomial, and $\mathcal{N}$ is $\mathcal{A}$-invariant. For $\alpha / \beta$ large enough, every matrix of the form (2.3) with $z \in B_{\delta}$ is a contraction, so $s^{-1} z^{(n)} s \in B_{\delta}$. But $s^{-1} w^{(n)} s$ will contain a non-zero entry multiplied by $\frac{\alpha}{\beta}$, so we achieve the claim.

Theorem 2.4. If $\phi$ is in $H^{\infty}\left(B_{\delta}\right)$, then $\forall z \in B_{\delta}$, we have $\phi(z) \in \operatorname{Alg}(z)$.

Proof: We can assume that $z \in B_{\delta}$ and that $\|\phi\| \leq 1$ on $B_{\delta}$. Let $w=\phi(z)$. If $w \notin \operatorname{Alg}(z)$, then by Lemma 2.2, there is an $s$ such that $s^{-1} z^{(n)} s \in B_{\delta}$ and $\left\|\phi\left(s^{-1} z^{(n)} s\right)\right\|=\left\|s^{-1} w^{(n)} s\right\|>1$, a contradiction.

Note that Theorem 2.4 does not hold for all nc-functions. In [4] it is shown that there is a class of nc-functions, called fat functions, for which the implicit function theorem holds, but Theorem 2.4 fails.

\section{Step II}

Let $F=\left\{x_{1}, \ldots, x_{N}\right\}$. Define $\lambda=x_{1} \oplus \cdots \oplus x_{N}$, and define $w=\phi\left(x_{1}\right) \oplus$ $\cdots \oplus \phi\left(x_{N}\right)$. As nc functions preserve direct sums (a consequence of being intertwining preserving) we need to find a function $\psi$ in $H_{1}^{\infty}\left(B_{\delta}\right)$ that has an nc-model, and satisfies $\psi(\lambda)=w$.

Let $\mathcal{P}_{d}$ denote the nc polynomials in $d$ variables, and define

$$
I_{\lambda}=\left\{q \in \mathcal{P}_{d}: q(\lambda)=0\right\} .
$$

Let

$$
V_{\lambda}=\left\{x \in \mathbb{M}^{d}: q(x)=0 \text { whenever } q \in I_{\lambda}\right\} .
$$

We need the following theorem from [3]:

Theorem 3.1. Let $\lambda \in B_{\delta} \cap \mathbb{M}_{n}^{d}$ and $w \in \mathbb{M}_{n}$. There exists a function $\psi$ in the closed unit ball of $H^{\infty}\left(B_{\delta}\right)$ such that $\psi(\lambda)=w$ if (i) $w \in \operatorname{Alg}(\lambda)$, so there exists $p \in \mathcal{P}_{d}$ such that $p(\lambda)=w$.

(ii) $\sup \left\{\|p(x)\|: x \in V_{\lambda} \cap B_{\delta}\right\} \leq 1$.

Moreover, if the conditions are satisfied, $\psi$ can be chosen to have a free realization.

Since $\phi(\lambda)=w$, by Theorem 2.4, there is a free polynomial $p$ such that $p(\lambda)=w$, so condition (i) is satisfied. To see condition (ii), note that for all $x \in V_{\lambda} \cap B_{\delta}$. we have $p(x)=\phi(x)$. Indeed, by Theorem 2.4, there is a 
polynomial $q$ so that $q(\lambda \oplus x)=\phi(\lambda \oplus x)$. Therefore $q(\lambda)=p(\lambda)$, so, since $x \in V_{\lambda}$, we also have $q(x)=p(x)$, and hence $p(x)=\phi(x)$. But $\phi$ is in the unit ball of $H_{1}^{\infty}\left(B_{\delta}\right)$, so $\|\phi(x)\| \leq 1$ for every $x$ in $B_{\delta}$.

So we can apply Theorem 3.1 to conclude that there is a function $\psi$ in $H^{\infty}\left(B_{\delta}\right)$ that has a free realization, and that agrees with $\phi$ on the finite set $F$.

Remark: The converse of Theorem 3.1 is also true. Given Theorem 2.4, the converse is almost immediate.

\section{Steps III and IV}

Let $\Lambda=\left\{x_{j}\right\}_{j=1}^{\infty}$ be a countable dense set in $B_{\delta}$. For each $k$, let $F_{k}=$ $\left\{x_{1}, \ldots, x_{k}\right\}$. By Step II, there is a function $\psi^{k} \in H_{1}^{\infty}\left(B_{\delta}\right)$ that has a free realization and agrees with $\phi$ on $F_{k}$. By Theorem 1.8, there exists a Hilbert space $\mathcal{M}^{k}$ and a $\mathcal{B}\left(\mathbb{C}, \mathcal{M}^{k} \otimes \mathbb{C}^{J}\right)$ valued nc-function $u^{k}$ on $B_{\delta}$ so that, for all $n$, for all $x, y \in B_{\delta} \cap \mathbb{M}_{n}^{d}$, we have

$$
1-\psi^{k}(y)^{*} \psi^{k}(x)=u^{k}(y)^{*}\left[\begin{array}{c}
\stackrel{1}{\otimes} \\
1-\delta(y)^{*} \delta(x)
\end{array}\right] u^{k}(x) .
$$

Embed each $\mathcal{M}^{k}$ in a common Hilbert space $\mathcal{H}$. Since the left-hand side of (4.1) is bounded, it follows that $u^{k}$ are locally bounded, so we can apply Theorem 1.9 to find a sequence of unitaries $U^{k}$ so that, after passing to a subsequence, $U^{k} * u^{k}$ converges to a function $v$ in $\operatorname{Hol}_{\mathcal{H}}^{\text {nc }}(\Omega)$. We have therefore that

$$
1-\phi(y)^{*} \phi(x)=v(y)^{*}\left[\begin{array}{c}
1 \\
1-\delta(y)^{*} \delta(x)
\end{array}\right] v(x)
$$

holds for all pairs $(x, y)$ that are both in $\Lambda \cap \mathbb{M}_{n}^{d}$ for any $n$, so by continuity, we get that (4.2) is an nc-model for $\phi$ on all $B_{\delta}$, completing Step III.

Finally, Step IV follows by applying Theorem 1.8.

\section{Closing remarks}

One can modify the argument to get a realization formula for $\mathcal{B}(\mathcal{K}, \mathcal{L})$-valued bounded nc-functions on $B_{\delta}$, or to prove Leech theorems (also called Toeplitzcorona theorems - see [10] and [8]). For finite-dimensional $\mathcal{K}$ and $\mathcal{L}$, this was done in [2]; for infinite-dimensional $\mathcal{K}$ and $\mathcal{L}$ the formula was proved in [6], using results from [7]. 


\section{References}

[1] J. Agler and J.E. McCarthy. Wandering Montel theorems for Hilbert space valued holomorphic functions. To appear. arXiv:1706:05376.

[2] J. Agler and J.E. McCarthy. Global holomorphic functions in several non-commuting variables. Canad. J. Math., 67(2):241-285, 2015.

[3] J. Agler and J.E. McCarthy. Pick interpolation for free holomorphic functions. Amer. J. Math., 137(6):1685-1701, 2015.

[4] Jim Agler and John E. McCarthy. The implicit function theorem and free algebraic sets. Trans. Amer. Math. Soc., 368(5):3157-3175, 2016.

[5] Sriram Balasubramanian. Toeplitz corona and the Douglas property for free functions. J. Math. Anal. Appl., 428(1):1-11, 2015.

[6] J.A. Ball, G. Marx, and V. Vinnikov. Interpolation and transfer function realization for the non-commutative Schur-Agler class. To appear. arXiv:1602.00762.

[7] Joseph A. Ball, Gregory Marx, and Victor Vinnikov. Noncommutative reproducing kernel Hilbert spaces. J. Funct. Anal., 271(7):1844-1920, 2016.

[8] M. A. Kaashoek and J. Rovnyak. On the preceding paper by R. B. Leech. Integral Equations Operator Theory, 78(1):75-77, 2014.

[9] Dmitry S. Kaliuzhnyi-Verbovetskyi and Victor Vinnikov. Foundations of free non-commutative function theory. AMS, Providence, 2014.

[10] Robert B. Leech. Factorization of analytic functions and operator inequalities. Integral Equations Operator Theory, 78(1):71-73, 2014.

[11] J.E. Pascoe and R. Tully-Doyle. Free Pick functions: representations, asymptotic behavior and matrix monotonicity in several noncommuting variables. arXiv:1309.1791. 